\title{
How to Detect Quantum (de Broglie) Waves
}

\author{
Carlos López \\ Department of Physics and Mathematics, Universidad de Alcalá de Henares (UAH), Alcalá de Henares, Spain \\ Email: carlos.lopez@uah.es
}

How to cite this paper: López, C. (2020) How to Detect Quantum (de Broglie) Waves. Open Access Library Journal, 7: e6741. https://doi.org/10.4236/oalib.1106741

Received: August 24, 2020

Accepted: September 18, 2020

Published: September 21, 2020

Copyright $\odot 2020$ by author(s) and Open Access Library Inc.

This work is licensed under the Creative Commons Attribution International License (CC BY 4.0).

http://creativecommons.org/licenses/by/4.0/

\begin{abstract}
A very simple (therefore strong) argument, grounded in the action reaction principle, predicts the existence of isolated quantum, de Broglie waves. In this article I propose an experimental set up able to detect these quantum waves. As far as quantum waves are associated to any kind of elementary particles, massive or massless, charged or neutral, etc., its character is probably gravitational, i.e., they are micro-gravitational waves. The quantum wave is isolated when a particle can follow two (or more) alternative paths which later on can rejoin and interfere. We know that the corpuscular particle follows one path while the wave follows both. This wave could perturb a laser beam, and this perturbation could be detected in a Mach-Zehnder interferometer.
\end{abstract}

\section{Subject Areas}

Modern Physics, Quantum Mechanics

\section{Keywords}

Quantum de Broglie Waves, The Action Reaction Principle in Quantum Mechanics

\section{Introduction}

In a few years we will celebrate the 100th anniversary of the publication [1] [2] by Louis de Broglie predicting the existence of physical waves associated to elementary particles (as, e.g., electrons) and other quantum systems (atoms, molecules). Very soon, Davisson [3] detected experimentally the diffraction of electrons by a crystal of nickel. The existence of isolated physical waves, that is, independent of the accompanying particle, does not belong to the orthodox interpretation of Quantum Mechanics. The so called wave-particle duality expresses the dual behaviour of elementary particles as corpuscles or waves, depending on the performed experiment or measurement. Obviously, this dual behaviour of a 
single system departs from any "classical" or rational interpretation.

However, an extremely simple scientific argument [4], grounded in the action reaction principle, predicts the existence of isolated physical waves, also denoted quantum or de Broglie waves. There are inconsistencies between the action-reaction principle and the projection rule under measurement of Quantum Mechanics [5] [6]. Whenever the trajectory of an elementary particle splits into two or more alternative paths, which later on can rejoin and interfere, we know that the corpuscular particle follows just one of the paths and therefore another wavelike system must follow both. Notice that if we block one of the paths the interference phenomenon disappears, there is a change of state associated to the interaction of the system (particle and wave) with the blocking element. Some change of state of the blocking system must happen too (action-reaction), but the corpuscular particle arriving at the junction of paths must follow the alternative path and cannot interact with the blocking system. Therefore another subsystem, the physical wave, must locally interact with the blocking element.

This is a rational description of the two-slit experiment (with, e.g., electrons), and of the self-interference of a photon in a Mach-Zehnder [7] [8] interferometer. In the first case, the electron goes through one slit while the associated physical wave follows both, so that if one slit is blocked, although the electron following the other slit arrives at the final screen, there is no more interference pattern on this final screen. Similarly, we can adjust the length of the arms of an interferometer in such a way that an incoming laser beam takes exclusively one of the two alternative exit gates. This happens even if the photons of the beam are injected one by one into the interferometer, that is, there is self-interference of the single photon. If we block one of the arms and the photon arrives at the exit gates it must have followed the other arm. But in absence of one of the wave components (the one which is blocked) there is no more self-interference and the photon can take both exit gates with equal probability.

We can also use a Stern-Gerlach device [9] to split the trajectory of an electron into two alternative paths, corresponding to spin up and down in the measured direction, orientation of the device. If we locate another device with opposite spatial orientation, and use an adequate electromagnetic field to redirect the spin up and down trajectories into the second device, we will reconstruct the initial spin state of the incoming electron, because of the superposition and interference of both spin components. Again, if we block one of the trajectories there is no more interference and the initial spin state is destroyed. Obviously, the electron arriving at the second Stern-Gerlach device has followed the unblocked trajectory; another system, the quantum wave, has followed the blocked path and locally interacts with the blocking system. In this reasoning we are systematically applying the action-reaction principle and locality of interactions, both well established principles in Physics.

\section{The Experimental Set Up}

We can use then a Stern-Gerlach device to split the trajectory of an isolated elec- 
tron, and try to detect the quantum wave in one of the outgoing trajectories (say, that of spin up) while the corpuscular electron is detected in the alternative (spin down) path. Similarly, we can use a beam splitter acting on an isolated incoming photon to split its trajectory into two, and try to detect the physical wave in one of the outgoing paths while the photon is detected in the other path.

As these physical, quantum waves are associated to all types of elementary particles, massless or massive, charged or neutral, etc., they must have a gravitational character, i.e., they must be some kind of microgravity waves [10] [11] [12]. These waves can then perturb the trajectory of a laser beam. If the incoming laser beam is injected in a Mach-Zehnder interferometer adjusted to get totally destructive interference in one exit gate (say A) and totally constructive in the other (B), once the laser beam is perturbed by intersection of one of the arms of the interferometer with the path of the quantum wave (either associated to an electron or to a photon, which follows another path) we should observe the perturbation by detecting some signal in the previously empty gate $A$. That is, the intersecting quantum wave perturbs, modifies the adjustment of the interferometer and there is (momentarily) no more totally destructive interference in gate A.

The experimental set up contains then in one side an incoming particle (photon or electron), with a splitter (beam splitter for photons or Stern-Gerlach device for electrons, Figure 1) and, in the other side a Mach-Zehnder interferometer with an incoming laser beam, Figure 2, adjusted to get totally destructive (respectively constructive) interference in exit gate A (resp. B). We locate both systems in such a way that one outgoing path of the elementary particle crosses one arm of the interferometer. We measure the arrival of the particle to some point of the alternative path, and if the particle has followed the alternative path (not the one crossing the arm of the interferometer) we expect to measure some signal in exit A of the interferometer, Figure 2, caused not by the corpuscular particle which has followed the alternative path but by the physical, quantum de Broglie wave. Obviously, when the particle follows the path which intersects the interferometer we could get some signal in A too, caused by the local interaction between the particle and the laser beam, but this case is not of our interest.

The first figure is an abstract representation of the trajectory of a particle (photon or electron) that splits into two paths (which later on could rejoin and interfere). The splitter will be a beam splitter for photons or a Stern-Gerlach device for electrons. The small black disk represents the intersection of one path with one arm of the Mach-Zehnder interferometer.

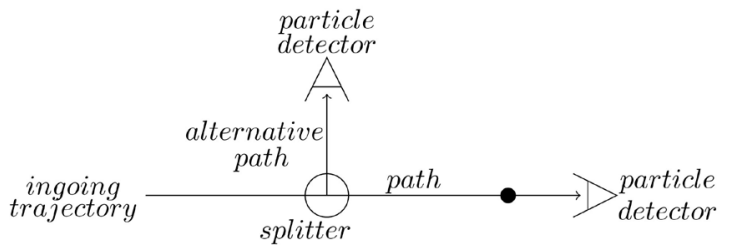

Figure 1. Ingoing trajectory of the particle, the splitter and two alternative outgoing paths. 


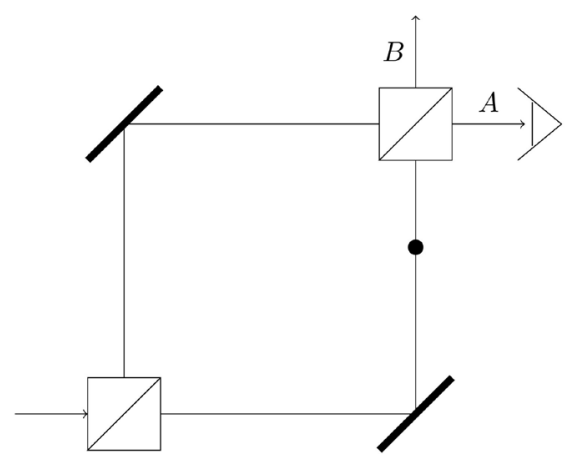

Figure 2. The Mach-Zehnder interferometer and an intensity detector in exit gate A.

Figure 2 represents the Mach-Zehnder interferometer, with its two beam splitters and its two mirrors. The arms lengths are adjusted in such a way that there is totally destructive interference in exit gate A. The small dark disk represents the intersection between that arm of the interferometer and one possible path of the particle.

Any perturbation over the beam along one arm of the interferometer will generate a small transient signal in exit $\mathrm{A}$. We expect that when the particle (photon or electron) follows the alternative path, the one which does not intersect the interferometer, we will detect the particle at the end of the alternative path (with the particle detector there located), and correlatively a signal in gate A. The signal is not caused by the particle, which follows the alternative path, but by the quantum wave which follows the path intersecting the interferometer. We will also detect some signal in A when the particle follows the path intersecting the interferometer, but this case is not of our interest, as far as it represents a local interaction between the particle and the laser beam.

\section{Summary}

The action reaction principle is not automatically fulfilled along quantum measurements. We analyse simple examples in which the action reaction principle is apparently violated. This happens when an elementary particle (photon or electron) goes through a splitter (beam splitter for photons or Stern-Gerlach device for electrons); the particle follows (stochastically) one outgoing path, while the associated quantum de Broglie wave follows both. We can try to detect the wave by intersecting its path with one arm of a Match-Zehnder interferometer, in such a way that it perturbs the length adjustment of the apparatus, and we could get some signal in the previously forbidden exit gate. The particle is detected on the alternative path, and we can study the correlation between wave and particle detections. If the particle follows the initial path crossing the interferometer we could get a signal too, but this case is not of our interest.

\section{Conflicts of Interest}

The author declares no conflicts of interest regarding the publication of this paper. 


\section{References}

[1] de Broglie, L. (1923) Waves and Quanta. Nature, 112, 540. https://doi.org/10.1038/112540a0

[2] de Broglie, L. (1924) Recherches sur la Th'eorie des Quanta. PhD Thesis, Masson, Paris.

[3] Davisson, C.J. (1928) The Diffraction of Electrons by a Crystal of Nickel. Bell System Technical Journal, 7, 90-105. https://doi.org/10.1002/j.1538-7305.1928.tb00342.x

[4] Lopez, C. (2020) De Broglie Waves. Open Access Library Journal, 7, e6100. https://doi.org/10.4236/oalib.1106100

[5] Lopez, C. (2017) Relativistic Locality and the Action Reaction Principle Predict de Broglie Fields. Quantum Studies: Mathematics and Foundations, 4, 149-157. https://doi.org/10.1007/s40509-016-0089-3

[6] Lopez, C. (2018) The Action Reaction Principle in Quantum Mechanics. In: Khrennikov, A. and Toni. B., Eds., Quantum Foundations, Probability and Information. 31-38. https://doi.org/10.1007/978-3-319-74971-6_4

[7] Zehnder, L. (1891) Ein neuer Interferenzrefraktor. Zeitschrift für Instrumentenkunde, 11, 275-285.

[8] Mach, L. (1892) Ueber einen Interferenzrefraktor. Zeitschrift für Instrumentenkunde, 12, 89-93.

[9] Gerlach, W. and Stern, O. (1922) Das magnetische Moment des Silber-atoms. Zeitschrift für Physik, 9, 353-355. https://doi.org/10.1007/BF01326984

[10] Feoli, A. (2011) The Amplitude of the de Broglie Gravitational Waves. Modern Physics Letter A, 24, 2497-2505. https://doi.org/10.1142/S0217732309031685

[11] Agavriloaei, N. (2012) Matter Waves in a Static Gravitational Field. Journal of Modern Physics, 3, 750-754. https://doi.org/10.4236/jmp.2012.38098

[12] Logiurato, F. (2014) Relativistic Derivations of de Broglie and Plank-Einstein Equations. Journal of Modern Physics, 5, 1-7. https://doi.org/10.4236/jmp.2014.51001 